\title{
TEMAS PARA LA INVESTIGACIÓN NEUROEDUCATIVA: ENFOQUE TRANSDISCIPLINARIO
}

\author{
Jorge Aurelio Rodriguez Huerta \\ Hugo Gonzalez Aguilar
}

\section{Resumen}

El presente artículo trata sobre la posibilidad de investigar e integrar las disciplinas de manera coherente: la neurociencia y la educación, desde un enfoque transdisciplinario, porque la realidad es compleja y no puede ser estudiada por disciplinas aisladas. Se propone temas para desarrollar en la integración de estas áreas y se concluye con una propuesta.

Palabras clave: Disciplina, educación, investigación, neurociencia, transdisciplinar

\section{Abstract}

This article discusses the possibility to investigate and integrate coherently disciplines: neuroscience and education from an interdisciplinary y approach because reality is complex and can not be studied as isolated disciplines. It is proposed to develop issues in integrating these areas and concludes with a proposed.

Keywords: Disciplinary, education, research, neuroscience, transdisciplinary 


\section{INTRODUCCIÓN}

En nuestro país, asi como ha predominado un paradigma (cuantitativo) en la investigación, lo mismo ha ocurrido con nuestro sistema educativo, que solo ha tratado los problemas de una manera aislada, cada quien resolviendo los problemas desde su área o desde su asignatura, pero no de manera integral, como debiera ser, ya que la educación (desde el nivel inicial hasta el sistema universitario) es un fenómeno complejo que requiere el estudio interrelacionado de varias disciplinas, o mejor dicho, a través de un enfoque transdisciplinar.

En este sentido, el presente estudio se enfoca en aspectos fundamentales que debe desarrollar la neurociencia en la educación, pero con un enfoque transdisciplinario. Primero, integrando los niveles educativos; segundo, integrando las universidades que tienen visiones y objetivos tan dispares como los objetivos de sus promotores o directores, la integración o la consideración de las comunidades nativas (que Peñaloza las consideró como las del cuarto mundo) en el proceso educativo; a continuación, viendo la integración de los diversos sectores para que se comprometan $\mathrm{y}$, por último, encarando los temas en investigación neuroeducativa con un enfoque transdisciplinar.

Aún no nos hemos integrado o unido como corresponde, seguimos viendo las brechas o diferencias abismales entre la educación urbana y rural; entre los colegios privados y públicos, al igual que nuestro sistema universitario. Por otro lado, falta integrar nuestra experiencia educativa o pedagógica que aún no se ha rescatado con las experiencias extraordinarias de transdisciplinariedad, como lo podemos comprobar con la praxis educativa de Encinas, Caro Ríos y el propio Arguedas. Debemos partir de sus experiencias exitosas, que primero la hicieron y luego la escribieron; un enfoque desde la práctica a la teoría y no a la inversa.

En el presente artículo, se trata de analizar los temas que se deben investigar y cómo se deben integrar las disciplinas para establecer un puente adecuado entre la neurociencia y la educación, desde un enfoque transdisciplinario.

\section{FUNDAMENTACIÓN}

\section{a) La desintegración del sistema educativo}

El sistema educativo peruano evidencia su desintegración a nivel de estudios y en la misma práctica. Esto se evidencia, entre el nivel secundario y universitario, por el número de academias preuniversitarias, e incluso los mal 1lamados colegios preuniversitarios, que se encargan de preparar a los estudiantes para su ingreso en las universidades. El colegio debe preparar suficientemente bien al estudiante para ingresar en las universidades; a las mismas universidades les corresponderá también reorientar sus exámenes en 
función de los conocimientos que dominan los estudiantes, o bajo los cuales han sido preparados.

Otro aspecto de desarticulación o desintegración se da entre las mismas universidades: cada una tiene una visión distinta, la mayoría centrada en las carreras de mayor rentabilidad más que en la formación social-integral del ser humano. En la práctica, se evidencia el predominio de formar profesionales para trabajar en las empresas y que estas den mayor rentabilidad. Por esta razón, pocos estudiantes quieran ser profesionales en arte, música, filosofia, literatura; simplemente no estudian porque no son rentables o no hay mayor campo de acción, o la empleabilidad para estas áreas es inexistente $\mathrm{y}$, sin existen, son muy poco remuneradas.

\section{b) La integración de la educación con los sectores sociales}

Es de notar que los sectores empresariales o civiles están poco involucrados con la educación; la gestióngerencia de las empresas se orienta muy poco a la educación de la sociedad. Los medios de comunicación de señal abierta funcionan como entes des educadores; pues en su mayoría difunden violencia o programas de entretenimiento con mensajes nada edificantes o formativos.

Se requiere una educación integradora que interactúe con los diversos sectores; una educación que ayude a construir "una sociedad integrada -fundada en el diálogo, el sentido de pertenencia y la solidaridad- y un Estado moderno, democrático y eficiente" (CNE, 2006, p. 28).

\section{c) E1 sistema educativo alejado de la ciencia}

El sistema educativo se ha visto como un ente que solo transmite información, sin ser analizada, pensada, razonada. Nuestra educación se asemeja a una sociedad de la ignorancia (Brey, 2009), porque no profundiza en el conocimiento científico o, mejor dicho, no desarrolla o discute el conocimiento científico, sino más bien un conocimiento utilitario y pragmático que se basa solo en el hacer. No se orienta a la innovación, a la generación de nuevos conocimientos. El sistema educativo se debe sustentar en teorías sólidas, como por ejemplo las teorias del aprendizaje centradas en la psicologia, las teorías didácticas centradas en orientar mejor el proceso de enseñanzaaprendizaje, tal como se plantea a través de la teoría de los procesos conscientes de Álvarez de Sayas (2004), que propone un proceso de aprendizaje científico que va desde la identificación del problema hasta la evaluación de los resultados de todo el proceso, pasando por el uso del método, de los medios, de las formas, objetivos, etc.

d) E1 sistema educativo al margen de la neurociencia

De lo referido en los puntos precedentes, se infiere que la educación es asistemática, informal, al 
estilo de la educación tradicional; la transmisión de conocimientos es sin discusión, sin análisis, sin crítica. Se requiere que los procesos de enseñanza-aprendizaje se realicen de manera científica, orientados a la innovación, a crear nuevos conocimientos en el contexto de lo que significa la praxis de la responsabilidad social.

Lo que se está enseñando y/o aprendiendo está al margen de la neuroeducación. Un primer aspecto relevante que falta es aclarar respecto a la tarea de las neurociencias, nos lo proporcionan De la Barrera y Donolo (2009), quienes sostienen:

La tarea central de las llamadas neurociencias es la de intentar explicar cómo es que actúan millones de células nerviosas individuales en el encéfalo para producir la conducta y cómo, a su vez, estas células están influidas por el medioambiente, incluyendo la conducta de otros individuos (Jessel, et al. 1997). Precisamente, las neurociencias están contribuyendo a una mayor comprensión, y en ocasiones a dar respuestas a cuestiones de gran interés para los educadores; por ejemplo, hay evidencias según lo muestran las investigaciones de que tanto un cerebro en desarrollo como uno ya maduro se alteran estructuralmente cuando ocurren los aprendizajes (Bransford, et al., 2003).

Otro aspecto importante: nos falta concebir la educación, desde la perspectiva de la neuroeducación, como "el sistema integrado de conocimientos biológicos, neurológicos y psicológicos cuyo objetivo es la comprensión y explicación de la estructura de la actividad neuropsíquica de la persona normal principalmente, incluyendo el desarrollo formativo del cerebro como soporte neuroactivo de la consciencia dentro del contexto histórico social en que le ha tocado vivir y desarrollarse" (Camacho, 2013, p. 3). En este sentido, se descuida por ejemplo el contexto real, muchas veces imitando otras realidades o soñando con otras realidades muy diferentes a la realidad peruana.

Falta concebir a la neurociencia como una disciplina transdisciplinar, porque esta interactúa con otras ciencias, como la psicología, la biología, la neurología, disciplinas que permitirian orientar mejor el sistema educativo, sobre todo para que se comprendan los procesos de asimilación o procesamiento de la información de una manera crítica y creativa, con libertad de pensamiento, sin ataduras, ni reglas que limiten a descubrir o encontrar la verdad. Lo cual, obviamente, es un proceso complejo como es el fenómeno educativo.

Falta precisar las situaciones epistemológicas y metodológicas de la neuroeducación. "El desarrollo humano y los procesos de aprendizaje son plausibles de ser estudiados considerando diferentes niveles de análisis: el biológico, el cognitivo y 
el comportamental. A su vez, cada uno contendría subniveles. Por ejemplo, el nivel de análisis biológico incluiría a otros, como el genético, el molecular, el celular, el de las conexiones entre células y el de los sistemas o redes neurales. Por su parte, el cognitivo y el comportamental incluirian los de la conducta individual, los comportamientos sociales en diferentes contextos de desarrollo, así como también en el nivel de la comunidad y la cultura" (Colombo, et al, 2010). Esto implicaría comprender la dinámica del desarrollo del ser humano, a fin de que este comprenda y reproduzca la cultura, la ciencia con más eficiencia, eficacia; es decir, efectividad.

\section{EL SISTEMA EDUCATIVO Y LOS TEMAS DE INVESTIGACIÓN NEU- ROEDUCATIVA}

De la Barrera y Donolo (2009), consideran que "las investigaciones han demostrado que durante el desarrollo de nuevas vías neurales (Doetsch, 2005 y Schinder, 2002), nuestras sinapsis cambian todo el tiempo y es así como recordamos una y otra experiencia o vivencia" (p. 4). Asimismo, conciben que los neurotransmisores (dopamina y acetilcolina) incrementen los aprendizajes de los estudiantes porque refuerzan la concentración y proporciona satisfacción.

Se debe postular una integración entre la educación y la investigación en neurociencia cognitiva (Ansari y Coch, 2006, citado por De la Ba- rrera y Donolo, 2009). Este sería uno de los temas centrales para investigar y ver de qué manera la neurociencia cognitiva mejoraría nuestro sistema educativo, porque se basaría en una ciencia integrada por la educación, el cerebro, la mente y el aprendizaje.

Se propone diez (10) temas sin que ello signifique una regla; ya que puede haber mejores propuestas:

\section{1) Neurociencias y su aplicabilidad} en el salón de clases (Macías y Ferra, 2011)

Se debe tener una visión integrada de aprendizaje, enseñanza y conocimiento, que son los fundamentos de la educación. Esto implica que para aprender se debe planificar lo que se va a enseñar, luego reflexionar si se cumplió o no, si se ha logrado un efectivo aprendizaje; solo entonces comprobaremos que el sujeto conoce. Se debe considerar el proceso de enseñanza: la relación aprendiz-contexto para el aprendizaje. El estudiante, incluido el docente, debe ser consciente de que aprende o desaprende de las disciplinas para solucionar problemas de su entorno. Se debe tener en cuenta el perfil del docente y del estudiante. Se debe tener en cuenta su perfil y sobre todo los roles que compete.

\section{2) La neurociencia y los siete sabe- res (Pizano, 2007)}

Permite identificar cómo el discente puede aprender de una manera más efectiva y el docente cómo debe diseñar $\mathrm{u}$ orientar la planificación de su clase, de esta manera se puede logar estudiantes más críticos, creativos e 
innovadores. No se excluye los cuatro pilares del conocimiento (Jaques Delors) y el quinto pilar (Transformarse uno mismo y la sociedad)

\section{3) Desarrollo evolutivo y destrezas neuropsicológicas (Brauer, 2010)}

$\mathrm{Su}$ objetivo es especificar el desarrollo de las destrezas sobre las que se sustenta el desarrollo humano.

\section{4) Cerebro-mente-conducta (García)}

La tarea de las Neurociencias es aportar explicaciones de la mente y conducta en términos de procesos del encéfalo. ¿Cómo actúan millones de células nerviosas que generan procesos mentales y comportamentales? Los resultados podrian aplicarse a la educación para que los estudiantes logren desarrollar sus competencias en la vida de una manera eficaz o eficiente.

\section{5) Neurociencia y aprendizaje}

Comprende la posibilidad de aprender relacionando experiencias para que el aprendizaje sea más efectivo. Permite la posibilidad de explorar y enriquecer el aprendizaje, puesto que si el estudiante está contento, entonces la información será aprendida con mayor facilidad. Es importante el estudio de las emociones, ya que conducen la memoria, lo cual significa que si las emociones son placenteras, el rechazo a la información novedosa será menor y, por ende, el aprendizaje más efectivo.

\section{6) Influencia de la neurociencia en la formación docente}

Se fundamenta en que la pedagogía, base para la formación de los docentes, busca intencionalmente la transformación de las estructuras de conciencia del ser humano. En consecuencia, se debe entender el proceso de construcción del conocimiento como un aspecto de lo mental (que incluye lo biológico y psicológico) y de lo cultural. Involucra el aporte de la neurociencia y su influencia en el trabajo de aula, lo cual implica su capacidad de educabilidad del estudiante que tiene un sentido complejo y amplio.

\section{7) Neurociencia y metacognición} (De la Barrera y Donolo)

Habria que reflexionar sobre los aportes de la neurociencia respecto a cómo se aprende, pero aplicando las estrategias de la metacognición antes, durante y después de la realización de una actividad académica. Esto llevará a planificar, monitorear, controlar o evaluar nuestras actividades de aprendizaje, haciendo de las actividades didácticas algo atractivo para el estudiante, pero sobre todo de actividades reflexivas en las que el estudiante sepa qué aprende, cómo aprende y sobre todo para qué aprende.

\section{8) La programación neurolingüísti- ca y su relación con el aprendizaje}

Tiene que ver con la forma de pensar sobre sus experiencias, pero a la vez esta las comunica. Toma en cuenta el pensamiento y el lenguaje. El primero permite reflexionar y el segundo dar a conocer esa reflexión a través del lenguaje para que sea conocida. De manera específica, tiene que ver con los planes y programas y cómo estos permiten orientar un aprendizaje óptimo en los estudiantes o personas. 


\section{9) Neuroeducación y didáctica}

Es importante revisar, analizar y, sobre todo, aplicar los postulados o procesos de la neuroeducación al proceso de enseñanza-aprendizaje desde sus principales aspectos: estrategias, medios y materiales, evaluación, etc. Desde la perspectiva de la neuroeducación y didáctica, se tendría que cambiar las formar de enseñar y aprender, las estrategias a emplear y los criterios e indicadores con los que se va a evaluar al estudiante.

\section{0) Neuroeducación y Tecnologias de Información y de las Comunica- ciones (TICs)}

Es responsabilidad de establecer la relación de causas-efectos de los TICs en el proceso enseñanza-aprendizaje, en la independencia del estudiante en el rol de innovación y la generación de nuevos conocimientos.

\section{PROPUESTA TRANSDISCIPLI- NARIA DE LA NEUROEDUCACIÓN}

La educación, como un fenómeno complejo, debe ser estudiada desde la perspectiva de la transdisciplinariedad porque "intenta ordenar articuladamente el conocimiento, coordinándolo y subordinándolo en una pirámide que permita considerar orgánicamente todas las ciencias. Busca que las relaciones entre las ciencias trasciendan en la integración de un conjunto con sentido y que pueda englobar... diversas disciplinas que le permitan interpretar la realidad y los fenómenos que se presuponen unitarios" (Tamayo, 2004, p. 74).
El estudio del fenómeno educativo con el enfoque de la neurociencia debe estudiarse desde la transdisciplinariedad, lo cual implica:

...la necesidad de aportar conocimientos sobre el cerebro, su anatomía, funcionamiento, desmitificando su exclusivo estudio para las ciencias de la salud, asi como la revisión de las diversas teorias, entre ellas: el Cerebro Triuno, la Bihemisferialidad, la Inteligencia Emocional, la Teoría Neuronal y como todas ellas guardan una intima relación con los procesos cognitivos y los avances tecnológicos propios de este tercer milenio donde la información y la telemática avanza a pasos acelerados, lo cual conlleva al docente a estar en un proceso permanente de actualización y búsqueda de nuevas estrategias que le permitan facilitar con mayor fluidez y creatividad (P de Aparicio, 2009).

El núcleo central de la pedagogía, o para ser más específicos, de la didáctica, que es el proceso de enseñanzaaprendizaje también debe ser considerado desde la transdisciplinariedad, que:

...incluye el concepto de conexiones y fusiones entre disciplinas completamente diferentes... por lo tanto necesitamos crear una nueva metodología y nuevas organizaciones, incluyendo un lenguaje común que haga posible trascender los limites que separan a las disciplinas. El concep- 
to de transdisciplinariedad no es estático, sino absolutamente dinámico... la ciencia del aprendizaje y la educación podría tornarse uno de los campos más importantes del siglo 21. (Koizumi, 2004, p. 440, citado por De la Barrera y Donolo, 2009).

La educación y la neurociencia, desde la perspectiva de la transdisciplinariedad, requieren de nexos o puentes. Los puentes principales se basan en las siguientes disciplinas: la psicología educativa, la psicología cognitiva, la neuropsicología, la neurociencia cognitiva y otros aspectos provenientes de la biología, la pedagogía y la psicología.

Otro aspecto relevante sobre los puentes para relacionar a la neurociencia y a la educación, desde la transdisciplinariedad, es que debe partir de las propuestas metodológicas (Colombo, et al, 2009), centradas en:

- Observación directa de constructos hipotéticos en el nivel de la activación neural.

- Validación de constructos hipotéticos en el nivel de análisis comportamental, a través de la aplicaciónde técnicas de imágenes cerebrales.

- Análisis estructural y funcional de las estructuras neurales como medio para inferir estructurasy funciones a nivel comportamental.

- Uso del conocimiento sobre el funcionamiento neural para identificar y evaluar diferentes teorías acerca del comportamiento en el ámbito educativo.

En consecuencia; construir el estudio de la neuroeducación, desde la transdisciplinariedad, que debe fundarse en las disciplinas: biológicas, psicológicas y pedagógico-didácticas, de tal manera que se integren y permitan mejorar los aprendizajes, mejorar la educación o formación de las personas $\mathrm{y}$, por consiguiente, mejorar las condiciones de vida.

\section{CONCLUSIONES}

- La educación peruana está desarticulada entre sus diversos niveles, e incluso también dentro de sus mismos niveles; en el básico regular entre colegios privados y públicos, de la urbe y rurales; en el sistema universitario, con visiones distintas.

- La educación no está integrada con los diversos sectores sociales.

- La educación peruana sigue desarrollándose alejada de la ciencia y, sobre todo, de las neurociencias

- La educación desde la neurociencia debe desarrollar temas relevantes, como: neurociencia y aprendizaje, neurociencia y los siete saberes, neurociencia y su aplicación al salón de clases, neurociencia en la formación docente, neuroeducación y didáctica, etc.

- La educación se debe desarrollar desde un enfoque transdisciplinar, en la que se integren las siguientes disciplinas: psicología educativa, psicología cognitiva, neuropsicología, neurociencia cognitiva, biologia, pedagogía y didáctica

- La educación debe orientarse y buscar la innovación, la generación de nuevos conocimientos. 


\section{REFERENCIAS BIBLIOGRÁFICAS}

Álvarez de Sayas, C. (2004). Didáctica de la Educación Superior. La Escuela en la Vida. Chiclayo: Universidad Nacional Pedro Ruiz Gallo.

Brauer, A. (2020). Desarrollo evolutivo y neurociencia. Ponencia disertada en: "Congreso de Educación Inicial". Quito: Universidad San Francisco de Quito.

Brey, A. et al. (2009). La sociedad de la ignorancia y otros ensayos. Madrid: Infomanía.

Camacho Chumioque, J. (2013). Neurociencias de la educación (Módulo). Lima: Escuela de Postgrado de la UNE

Consejo Nacional de Educación. (2006). Proyecto Educativo Nacional al 2021. La educación que queremos para el Perú. Lima.

Colombo, J. et al. (2009). Neurociencia y educación: hacia la construcción de puentes interactivos. Buenos Aires.

De la Barrera, M. y Donolo, Danilo. (2009). Neurociencias y su importancia en contextos de aprendizaje. Revista Digital Universitaria. Volumen Nro. 10, ISSN: 1067-6079. http://www.revista.unam.mx/vol.10/num4/art20/int20.htm.

García García, E. Neurociencia: Aprendizaje y Educación. Madrid: Universidad Complutense.

Macias, J. y Ferra, R. (2011). Neurociencia y educación diferenciada... En búsqueda de la eficacia y equidad. XII Congreso Internacional de Teoría de la Educación. Universidad de Barcelona.

Miranda Esquerre, L. (2006). El problema de la enseñanza del español en el Perú. Lima: Editorial Ricardo Palma.

Morín, E. (1999). Los siete saberes necesarios para la educación del futuro. Francia: UNESCO.

P. de Aparicio, X. (2009). Neurociencias y la transdisciplinariedad en educación. En Revista Universitaria de Investigación y Diálogo Académico. Vol. 5, Nro. 2.

Pizano Chávez, G. (2007). La neurociencia y los siete saberes: La fuerza del futuro. Revista de Investigación Educativa, vol. 11, Nro. 20, 21-32. Julio-diciembre. Lima.

Tamayo y Tamayo, M. (2004). El proceso de la investigación científica. Cuarta Edición. México: LIMUSA. 